\title{
DISCUSSION
}

\section{Trends in large-deformation analysis of landslide mass movements with particular emphasis on the material point method}

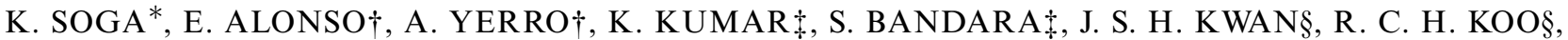 \\ R. P. H. LAW§, J. YIU\|, E. H. Y. SZE§ and K. K. S. HO§
}

\begin{abstract}
Contribution by J. S. H. Kwan, R. C. H. Koo, R.P.H. Law, J. Yiu, E. H. Y. Sze and K. K. S. Ho

Soga et al. (2016) examined a number of continuum-based large-deformation analysis methods to simulate the complex mechanics of landslide motion. In particular, the authors highlighted the benefits of the material point method (MPM) and presented two case studies to demonstrate its capability in modelling the pre- and post-failure behaviour of landslides. Some interesting findings were obtained. The authors mentioned that further work is required for practitioners to gain confidence in adopting these numerical tools in engineering practice. The need for verification of numerical models against case studies and investigation of constitutive models was emphasised.

The discussion contributors concur with the authors' views and agree that the use of numerical models in landslide simulation plays an important role in engineering practice, especially for quantified design of risk assessment and landslide hazard mitigation measures. In this regard, the discussion contributors would like to make their contribution to this subject by presenting the latest development of landslide mobility modelling in Hong Kong. For practical application, the landslide mobility model has been coupled with dynamic structural analysis to facilitate the design of landslide mitigation measures. Advances in the simulation of debris-barrier interaction are also discussed.
\end{abstract}

\section{VERIFIED LANDSLIDE MODELS}

The various analysis methods introduced by the authors have previously been adopted by the discussion contributors to simulate the landslide runout dynamics (Kwan \& Sun, 2007; Yiu et al., 2012; Huang et al., 2014; Kwan et al., 2015; Law et al., 2016). Despite the limitations of these methods as discussed by the authors, the models were successfully calibrated against notable landslide case histories in Hong Kong and laboratory flume tests with promising results. A brief summary of the discussion contributors' experience is given below.

Kwan \& Sun (2007) used the particle-in-cell (PIC) method to develop a landslide runout simulation model termed the '3D debris mobility model'. The model has been successfully benchmarked against a wide spectrum of landslide

\footnotetext{
* University of California, Berkeley, CA, USA.

$\uparrow$ Polytechnic University of Catalonia, Barcelona, Spain.

* Department of Engineering, University of Cambridge, Cambridge, UK.

§ Geotechnical Engineering Office, Civil Engineering and Development Department, Hong Kong SAR Government, Hong Kong, P. R. China.

|| Ove Arup and Partners Hong Kong Ltd, Hong Kong, P. R. China.
}

cases involving a failure volume ranging from $1000 \mathrm{~m}^{3}$ to $26000 \mathrm{~m}^{3}$.

Yiu et al. (2012), Huang et al. (2014) and Kwan et al. (2015) have demonstrated that the use of the arbitrary Lagrangian-Eulerian (ALE) method in the threedimensional finite-element package 'Livermore Software Dial-a-yield non-linear analysis' (LS-Dyna) is a promising tool for modelling debris flow. The landslide mass was modelled using the Drucker-Prager yield criterion (Drucker \& Prager, 1952) taking advantage, for example, of its more reliable simulation of sudden changes in internal velocity profile of the debris flow (Crosta et al., 2003), and given its less demanding requirement of input parameters as compared with other complicated constitutive models. The above model has also been benchmarked against laboratory flume tests and actual landslide cases (Yiu et al., 2012).

Law et al. (2016) adopted the discrete-element method (DEM) to study the interaction between granular debris flow and baffles. The DEM model has been calibrated using a series of flume experiments reported by Choi et al. (2015). In this numerical model, resistances to relative translational and rotational motions between the discrete elements were induced by contact friction in terms of translational friction and a rolling resistance term. The rolling resistance was calculated using a directional constant torque model, which applied a constant torque on a particle to represent the rolling friction, as elaborated by Ai et al. (2011). The plastic deformation between the particles was controlled using the coefficient of restitution.

\section{PRACTICALITY OF LANDSLIDE MOTION SIMULATION}

The above-cited studies have demonstrated the capability of various calibrated numerical methods in simulating the landslide motion. The calibrated models have been applied in geotechnical design practice in Hong Kong. Pre-failure behaviour and failure initiation are not modelled, as this is not normally needed, especially for natural terrain (Ho \& Roberts, 2016). The total stress approach is typically adopted in the models (Kwan \& Sun, 2007; Yiu et al., 2012; Huang et al., 2014; Kwan et al., 2015) in order to reduce the number of parameters required. This simplification echoes the authors' concerns about reducing numerical errors and improving computational efficiency. At the same time, it meets the needs of the geotechnical engineering practice with sufficient resolutions. In Hong Kong, landslide motion modelling is an important step to facilitate rational design of natural terrain landslide hazard mitigation measures. For this purpose, it would be pragmatic to focus on the post-failure motion rather than failure initiation in the simulation. In practice, proper modelling of failure initiation is not straightforward, as many of the landslide-triggering factors such as ground variability and complex hydrogeological condition (e.g. erosion pipes) are uncertain and 
cannot be explicitly and confidently accounted for in the models.

For post-failure modelling, the importance of pore water pressure would be controlled by the integrity of the skeleton of soil particles. When the landslide debris has mixed with an abundant amount of surface water and the soil particle skeleton is largely destroyed, the actual generation of excess pore water pressure will be very complex and the complicated effective stress models may have a low resolution and large uncertainties, whereas a total stress model suitably calibrated against good quality data will suffice in routine practice.

\section{COUPLED ANALYSIS OF DEBRIS-BARRIER INTERACTION}

One of the primary goals of the dynamic simulation of landslides is to facilitate the design of mitigation measures such as reinforced concrete rigid barriers and steel flexible barriers. In the conventional approach, landslide motion analysis and structural analysis of the barrier are carried out separately. The landslide motion is first simulated to obtain design parameters such as the flow velocity and depth, which are then converted into a pseudo-static impact force as input to a separate structural model. This approach neglects the dynamics of debris-barrier interaction and may misrepresent the actual barrier behaviour.

The discussers have recently conducted coupled analysis to simulate the dynamic interaction between landslide debris and a flexible barrier structure using LS-Dyna. The structural model was combined with the landslide mobility model to form a 'numerical flume'. Most of the structural elements of the flexible barrier were explicitly modelled and the non-linearity of energy dissipation elements was incorporated. The numerical flume model was successfully verified against a large-scale instrumented field test in Illgraben, Switzerland. It was found that the model was capable of simulating the impact of debris flow on the flexible barrier, and successfully reproduced the subsequent deformation of the barrier and its structural members. It also managed to reasonably replicate the forces developed in various structural components and gave the maximum debris impact pressure exerted on the barrier netting. The LS-Dyna model, based on the ALE method, appears to provide an effective tool to simulate the debris-structure interaction in addition to the landslide debris mobility, hence providing insights regarding the actual behavioural mechanism. This would greatly assist with the rational design of the landslide mitigation measures.

\footnotetext{
Authors' reply

The authors thank the discussion contributors for their contribution. The authors agree with the contributors' view that the use of numerical models in landslide simulation plays an important role in engineering practice. As demonstrated in their contribution, the Hong Kong government has been one of the front runners in the world in the area of large-deformation modelling of landslides. They have been developing both continuum based and discrete-element based computational tools to assess the risk of landslides in Hong Kong and to evaluate the feasibility of various hazard mitigation measures that they have designed and implemented. Their effort in applying such computational tools to engineering practice should be acknowledged.
}

They state that pore water pressure development is important in understanding the post-failure behaviour of landslides. However, they argue that the process of excess pore water pressure generation is very complex and hence at present they prefer to use a total stress model calibrated against good-quality data. The authors agree that this is the state-of-the art engineering practice in post-failure assessment of landslides. In the paper under discussion, however, the authors presented some effective stress-based soil-pore water coupled analysis methods for the large-deformation modelling of geomaterials and the two landslide case studies were used to demonstrate the ability of such methods and their future potential. The authors believe that such analysis will provide further insights into understanding the complex soil and pore water interaction behaviour and will move the profession to engage proactively not only in conventional pre-failure assessment but also in post-failure assessment of landslide problems.

\section{REFERENCES}

Ai J., Chen J. F., Rotter J. M. \& Ooi J. Y. (2011). Assessment of rolling resistance models in discrete element simulations. Powder Technol. 206, No. 3, 269-282.

Choi C. E., Ng C. W. W., Song D., Law R. P. H., Kwan J. H. S. \& Ho K. K. S. (2015). A computational investigation of baffle configuration on the impedance of channelized debris flow. Can. Geotech. J. 52, No. 2, 182-197.

Crosta G. B., Imposimato S. \& Roddeman D. G. (2003). Numerical modelling of large landslides stability and runout. Natural Hazards Earth Syst. Sci. 3, No. 6, 523-538.

Drucker D. C. \& Prager W. (1952). Soil mechanics and plastic analysis for limit design. Q. Appl. Math. 10, No. 2, $157-165$.

Ho H. Y. \& Roberts K. J. (2016). Guidelines for natural terrain hazard studies, 2nd edn, GEO Report No. 138. Hong Kong, P. R. China: Geotechnical Engineering Office, Civil Engineering and Development Department, Government of the Hong Kong Special Administrative Regions.

Huang Y., Yiu J., Pappin J., Sturt R., Kwan J. S. H. \& Ho K. K. S. (2014). Numerical investigation of landslide mobility and debris-resistant flexible barrier with LS-DYNA. Proceedings of the 13th international LS-DYNA users conference, Dearborn, MI, USA, pp. 1-12.

Kwan J. S. H. \& Sun H. W. (2007). Benchmarking exercise on landslide mobility modelling - runout analyses using 3dDMM. In Proceedings of the 2007 international forum on landslide disaster management (eds K. Ho and V. Li), vol. 2, pp. 945-966. Hong Kong, P. R. China: Geotechnical Division, Hong Kong Institution of Engineers.

Kwan J. S. H., Koo R. C. H. \& Ng C. W. W. (2015). Landslide mobility analysis for design of multiple debris-resisting barriers. Can. Geotech. J. 52, No. 9, 1345-1359.

Law R. P. H., Choi C. E. \& Ng C. W. W. (2016). Discrete-element investigation of influence of granular debris flow baffles on rigid barrier impact. Can. Geotech. J. 53, No. 1, 179-185.

Soga K., Alonso E., Yerro A., Kumar K. \& Bandara S. (2016). Trends in large-deformation analysis of landslide mass movements with particular emphasis on the material point method. Géotechnique 66, No. 3, 248-273, http://dx.doi.org/10.1680/ jgeot.15.LM.005.

Yiu J., Pappin J., Stuart R., Kwan J. S. H. \& Ho K. K. S. (2012). Landslide mobility and flexible barrier modelling using LS-DYNA. In Proceedings of the one day seminar on natural terrain hazard mitigation measures (eds C. K. Lau, E. Chan and J. Kwan), pp. 67-77. Hong Kong, P. R. China: The Association of Geotechnical and Geoenvironmental Specialists (Hong Kong) Limited. 